Preprint of: $A b$ initio and classical atomistic modelling of structure and defects in crystalline orthorhombic polyethylene: twin boundaries, slip interfaces, and nature of barriers

By: Olsson, Pär A. T.: Schröder, Elsebeth: Hyldgaard, Per: Kroon, Martin: Andreasson, Eskil: Bergvall, Erik.

Published in:

Polymer (2017), Volume 121, Pages 234-246.

DOI:

10.1016/j.polymer.2017.06.008

Published: 2017-06-22

Citation for published version:

Olsson, P. A. T., Schröder, E., Hyldgaard, P., Kroon, M., Andreasson, E. \& Bergvall, E. (2017). Ab initio and classical atomistic modelling of structure and defects in crystalline orthorhombic polyethylene: twin boundaries, slip interfaces, and nature of barriers. Polymer, 121, 234-246.

Link to published paper:

http://www.sciencedirect.com/science/article/pii/S0032386117305682 



\title{
$A b$ initio and classical atomistic modelling of structure and defects in crystalline orthorhombic polyethylene: twin boundaries, slip interfaces, and nature of barriers
}

\author{
Pär A. T. Olsson ${ }^{\mathrm{a}, *}$, Elsebeth Schröder ${ }^{\mathrm{b}}$, Per Hyldgaard ${ }^{\mathrm{b}, \mathrm{a}}$, Martin Kroon ${ }^{\mathrm{c}}$, Eskil Andreasson ${ }^{\mathrm{d}, \mathrm{e}}$, Erik Bergvall ${ }^{\mathrm{d}}$ \\ ${ }^{a}$ Materials Science and Applied Mathematics, Malmö University, SE-205 06 Malmö, Sweden \\ ${ }^{b}$ Department of Microtechnology and Nanoscience (MC2), Chalmers University of Technology, SE-412 96 Gothenburg, Sweden \\ ${ }^{c}$ Department of Mechanical Engineering, Linnaeus University, SE-351 06 Växjö, Sweden \\ ${ }^{d}$ Tetra Pak, Ruben Rausings gata, SE-221 86 Lund, Sweden \\ ${ }^{e}$ Department of Mechanical Engineering, Blekinge Institute of Technology, SE-371 79, Karlskrona, Sweden
}

\begin{abstract}
We study the stability of twin boundaries and slip in crystalline orthorhombic polyethylene by means of density functional theory (DFT), using a nonempirical, truly nonlocal density function, and by means of classical molecular dynamics (MD). The results show that, in accordance with experimental observations, there is a clear preference to chain slip over transverse slip for all considered slip planes. The activation energy for pure chain slip lies in the range $10-20 \mathrm{~mJ} / \mathrm{m}^{2}$, while that for transverse slip corresponds to $40-280$ $\mathrm{mJ} / \mathrm{m}^{2}$. For the (110)-slip plane the energy landscape is non-convex with multiple potential energy minima, indicating the presence of stable stacking faults. This suggests that dissociation of perfect dislocations into partials may occur. For the two low-energy twin boundaries considered in this work, $\{110\}$ and $\{310\}$, we find that the former is more stable than the latter, with ground state energies corresponding to 8.9 and $28 \mathrm{~mJ} / \mathrm{m}^{2}$, respectively. We have also evaluated how well the empirical MD simulations with the all-atom optimized potential for liquid MD simulations (OPLS-AA) and the coarse-grained united atom (UA) potential concur with the DFT results. It is found that an all-atom potential is necessary to partially capture the $\gamma$-surface energy landscapes obtained from the DFT calculations. The OPLS-AA predicts chain slip activation energies comparable with DFT data, while the transverse slip energy thresholds are low in comparison, which is attributed to weak close ranged monomer repulsion. Finally, we find that the $\mathrm{H}-\mathrm{H}$ interaction dominates the slip activation. While not explicitly represented in the UA potential, its key role is revealed by correlating the DFT energy landscape with changes in the electron distributions and by MD simulations in which components of the OPLS-AA intermolecular potential are selectively silenced.
\end{abstract}

Keywords: Polyethylene, Atomistic modelling, Slip

\section{Introduction}

High-density linear polyethylene (PE) commonly has a semicrystalline microstructure with high-density crystalline lamellae that are separated by amorphous regions having lower densities. The lamellae structures are commonly lozenge shaped crystals with the chains ordered by the orthorhombic unit cell structure $(a \approx 7.4 \AA, b \approx 4.9 \AA$ and $c \approx 2.5 \AA$ at room temperature) having $\{110\}$-faces terminating the sides of the crystal, while the lamella normal direction usually is parallel with the $\langle 001\rangle$-directions. The lamellae chains are folded back and forth multiple times, with the planar zig-zag polymer molecular chains mainly aligned parallel with the orthorhombic $c$-axis. This suggests that the bonding is characterized by strong covalent bonding in the $c$-direction, while van der Waals ( $\mathrm{vdW}$ ) interaction dominates the bonding in the perpendicular directions. The polymer is held together through the amorphous regions via tie-molecules that extend from one lamella to the neighboring and possibly even further. The density of the polymer and the size of the crystalline lamellae typically depend on

\footnotetext{
${ }^{*}$ Corresponding author

Email address: Par.0lsson@mah.se (Pär A. T. Olsson)
}

the manufacturing technique and process timings, but in general the thickness lies in the range 3-25 $\mathrm{nm}$ while the lateral dimensions of the lozenge shaped crystal can reach up to $50 \mu \mathrm{m}$ [1-3].

The plasticity of crystalline PE occurs mainly through three mechanisms: (i) slip [2-13], (ii) twinning [2, 7, 14-16] and (iii) martensitic transformation [2, 10, 17], ordered after frequency of occurrence. The most important modes of plastic deformation are the crystallographic slip mechanisms that do not break the polymer chains. This involves shearing either parallel or perpendicular to the [001]-direction, which are commonly referred to as chain slip and transverse slip, respectively. The former of these include slip for which the relative displacements occur along the chain direction, i.e. along the $c$ axis of the orthorhombic cell [3]. This implies the activation of the $(h k 0)$ [001]-type of slip system that is characterized by the [001]-Burgers vector, which is the shortest for the system, while transverse slip occurs on the $(h k 0)[k \bar{h} 0]$ system.

Experimental works have elucidated that chain slip is the sole plasticity mechanism at low temperature [5]. It proceeds mainly through the (100)[001] and (010)[001]-slip systems, where the former exhibits the least resistance $[3,4,6,9,12]$. Transverse slip has been found to occur primarily by the (100)[010] and 
(010)[100]-slip systems [7, 12], with a preference of the former since (100) is the easiest plane for slip to occur [3]. But there have also been reports of transverse slip on $\{110\}$ planes. Specifically, transverse slip of the $\{110\}\langle 1 \overline{1} 0\rangle$ type has been observed in the polar regions of spherulites [13], despite the size of the Burgers vector exceeding those of the [010] and [100]Burgers vectors. To facilitate such slip it was argued by Frank et al. [7], and later experimentally verified by Holland [8], that dissociation into partial dislocations through the reaction

$$
\langle 110\rangle \rightarrow\left\langle\frac{1}{2} \frac{1}{2} \kappa\right\rangle+\left\langle\frac{1}{2} \frac{1}{2} \bar{\kappa}\right\rangle
$$

may occur, resulting in reduced Burgers vectors. However, for the partials to have a smaller magnitude than that of [010] it is necessary for $\kappa$ to be small. This was investigated closer by Bacon and co-workers in a series of publications dealing with atomistic modelling of crystalline polyethylene [18-20]. By the employment of a relatively simple rigid covalent bond model, supplemented by a hybrid Buckingham and Born-Mayer potential to account for vdW interaction and repulsion, they found that stable stacking faults for $\kappa \approx 0$ and 0.5 were found to exist, indicating possible dissociation [19].

Twinning in PE was first recognized by Frank et al. [7] by the $\{110\}$ and $\{310\}$ modes, which are the two most plausible twinning mechanisms for plastic deformation, resulting in $\{110\}$ and $\{310\}$ twin boundaries. Based on geometric considerations it can be deduced that these modes have the smallest shear strains possible for twinning in orthorhombic PE [14] and they are the only ones that have been observed experimentally [15].

This paper seeks progress in two key stages of modelling the nature and crystal plasticity of the PE system, itself a typical example of a soft- or sparse-matter problem [21, 22]. Firstly we report a thorough $a b$ initio, or first principles, density functional theory (DFT) study using the vdW density functional (vdW-DF) method [23-30]. We compute the structure and cohesion of the crystal and the energies related to chain and transverse slip as well as to the formation of twin boundaries, using the recent consistent-exchange vdW-DF-cx formulation of the method [31]. There are formal arguments to expect a high degree of transferability, also when materials (like PE) combine regions with both dense and sparse electron concentrations [27-29]. Our finding of an excellent agreement between experimental and vdW-DF-cx characterizations of the structure and elastic response of the PE crystal strongly suggests that vdWDF-cx also accurately characterizes the PE defect properties as well as the stability and relaxation of twin boundaries. In effect, the $a b$ initio vdW-DF-cx results leverage broader insight into the nature of stacking faults and slip activation and can thus deepen the understanding of macroscopic properties of PE.

Secondly, we report a detailed assessment and discussion of performance for typical classical molecular dynamics (MD) modelling approaches for this PE sparse-matter problem. There are several interatomic potentials with different degrees of atomic resolution available in the literature that can be employed for classical MD modelling. Force fields for both allatom and coarse grained representations can be utilized depending on the modelling purpose. Specifically, in the present work we consider both the all-atom optimized potential for liquid simulations (OPLS-AA) and the coarse-grained unitedatom (UA) force field. These force fields display different levels of atomic resolution and are commonly used for polymer modelling. Although there is a multitude of all-atom potentials available in the literature, the former was chosen for the present paper based on its common use, its simplicity and relatively low computational cost, while the latter was chosen because it has been used extensively for investigating the mechanical properties of amorphous and semi-crystalline polymers [32-42].

It is important to seek proper validation and review their performance before using classical MD for more comprehensive modelling of the plastic behavior of PE systems. While the $a b$ initio vdW-DF-cx description provides a rich account of vdW forces, including a characterization of multipole and image-plane effects [23, 28, 43-45], typical classical MD approaches (including the herein utilized OPLS-AA and UA descriptions) describe vdW forces via a Lennard-Jones (L-J) term, which cannot be expected to hold over different binding lengths $[43,46,47]$. The empirical and parametric nature of the fitted classical MD potentials typically limits their transferability outside the fitting database. Hence, there is a priori no reason to expect that one set of classical MD parameters can describe both a perfect PE crystal and various PE defects unless they have been explicitely fitted to do so. Noting that empirical data for such classical MD validation is sparse in the literature, this paper exploits our confidence in the vdW-DF-cx results for this necessary assessment of classical MD.

The rest of the paper is organized as follows. In Sec. 2 we give an account of the necessary theory and present the utilized modelling tools, while in Sec. 3 we introduce the simulation setups and provide computational details. Results are presented and discussed in Sec. 4, while Sec. 5 contains our analysis of the nature of the slip activation barriers. Finally Sec. 6 contains our summary and conclusions.

\section{Theory}

\subsection{Ab initio density functional theory}

$A b$ initio DFT brings tremendous advantages to materials modelling in terms of accuracy, reliability, and in making system-specific characterizations and predictions [48, 49]. For a detailed computational materials study, the focus is on the electron behavior; the presence of nuclei can essentially be handled by inclusion of an external potential for electron dynamics. That still appears to be an impossible problem because the electron-electron interaction makes all attempts at direct calculations essentially futile for extended systems. However, the development of $a b$ initio DFT overcame this challenge and is today a great enabler of materials theory [48]. In essence, use of DFT allows us to deliver an, in principle, exact solution for the ground state density and energy at reasonable computational costs. DFT succeeds by rigorously mapping the physical problems onto an equivalent fictitious problem in which a set of quasi particles (with properties reflecting the actual electron properties) move in some effective potential but having no mutual interaction. 
The key step in securing accurate DFT characterization lies in designing good approximations for the exchange and correlation energy $E_{\mathrm{xc}}$, a functional whose value reflects the spatial variation in the electron density, $n(\mathbf{r})$. A long-standing challenge was capturing also the $\mathrm{vdW}$ forces that are ubiquitous in sparse-matter problems and, for example, provide cohesion between the PE macromolecules [21]. The Chalmers-Rutgers vdW-DF method [23-25, 29, 30] permits us to craft computationally efficient [50-52], truly nonlocal functional approximations [25, 31, 53] for $E_{\mathrm{xc}}$, capturing general sparse-matter interactions while building from a many-particle theory analysis of screening in the electron gas [30, 48, 54-57]. Complying with known constraints [24, 26, 28, 53, 58], the method provides a direct determination of $\mathrm{vdW}$ forces in the Rapcewicz-Ashcroft picture $[28,59]$. That is, the vdW-DF versions describe vdW forces as they naturally emerge in the presence of the screening defined by the surrounding electron gas [28, 30, 59]. This feature is important since weak and stronger forces generally compete $[27,30]$.

Our DFT exploration of the PE system is based on the recent consistent-exchange vdW-DF-cx version [27, 31]. Being strictly parameter-free and anchored directly in the electrondensity variation, it has the right mechanism for retaining an accurate characterization across a range of molecular separations $[43,46]$, for example, as relevant for the theoretical characterization of the PE defect systems. Also, the vdW-DF-cx version is the one most closely related to the formal many-particle theory specification [54-56] of $E_{\mathrm{xc}}$ since it uses the same analysis of the screened response for setting the balance between exchange and correlation terms [27, 28, 30, 31]. In light of these characteristics, the vdW-DF-cx version is a candidate for serving as a first truly general-purpose functional, and this version has already been proven to deliver highly accurate and transferable accounts of material structure, cohesion and excitations in many different types of extended and molecular systems, including molecular crystals [60, 61], layered materials [62-64], surfaces [65, 66] and transition metals [58]. We trust that it works equally well under general polymer configurations, both those of a perfect PE crystal and those of PE defects.

\subsection{Classical molecular dynamics}

We also seek to evaluate the accuracy and transferability of classical MD descriptions. These are formulations in which the atoms, that is, ions with their surrounding electron cloud, are described as composite objects that have some effective mutual interaction. The effective interaction reflects, for example, steric hindrance, covalent binding and vdW forces, for which the latter are typically described by a L-J term.

Classical MD simulations are highly desirable as a modelling tool because they are more computationally effective than $a b$ initio DFT and can track dynamical effects for larger systems and over much longer time scales. The effective potentials can be set by empirical input or from $a b$ initio calculations. On the one hand classical MD holds the advantage in that there is no need to compute the electron density. On the other hand, generic classical MD approaches cannot fully reflect interaction changes that occur as the electron distribution changes with

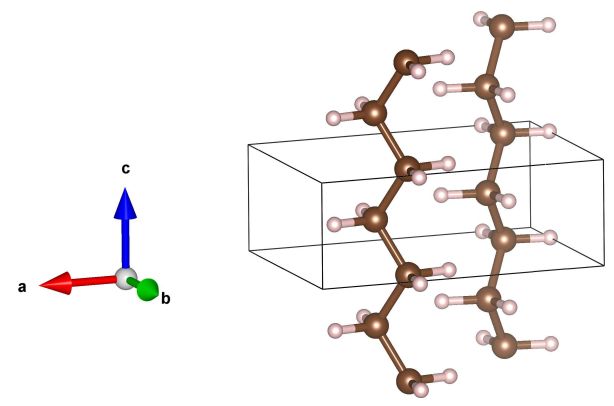

Figure 1: Illustration of the orthorhombic PE unit cell generated using the VESTA software [67]. The coordinate axes represent the directions of the lattice parameters $a, b$ and $c$.

binding or such that result from multipole effects [23, 43-45]. Such physics cannot be accounted for when using a L-J term to account for vdW forces. Classical MD descriptions must therefore be carefully validated for systems that are relevant to the intended applications.

\section{Simulation setup and computational details}

\subsection{Orthorhombic polyethylene}

There are mainly two types of crystalline phases that have been observed in semi-crystalline PE: orthorhombic and monoclinic crystals $[1,2]$. However, the monoclinic phase only occurs as a result of partial transformation of the orthorhombic crystal when subjected to high stress conditions, such as cold working and compression of drawn samples, or during melt crystallization under high pressure. Moreover, during annealing the monoclinic phase transforms back to the orthorhombic [68]. Thus, for most practical applications the orthorhombic phase is that of highest importance, which is why it is the only bulk phase considered in the present work, see Figure 1.

The orthorhombic unit cell contains four $\mathrm{CH}_{2}$-units and to find its optimal lattice parameters we adopt a two-step strategy. First we optimize the $c$-parameter, which is parallel to the chain direction, see Figure 1 . This can be done separately using two reasonable values of $a$ and $b$, because the bond strength is roughly two orders of magnitude stronger than in the $a$ and $b$-directions, which are characterized by weak vdW bonding. Once $c$ is found, we vary $a$ and $b$ while letting the atomic coordinates relax such that they are in equilibrium. By identifying the potential energy minimum, the optimal lattice parameters and atomic coordinates of the unit cell are found.

\subsection{Twin boundaries}

The two twin boundaries considered in the present work correspond to the $\{110\}$ and $\{310\}$ twins, see Figure 2. They form when the orthorhombic crystal is subjected to shearing and are those commonly observed experimentally. They are created through the rotation of the crystal about the [001]-axis with rotation angles corresponding to $-67^{\circ}$ and $55^{\circ}$ [2], respectively. To compute the twin boundary energy it is necessary to find the lowest energy interface. To this end, we consider highly symmetric configurations for each twin boundary. Primarily 


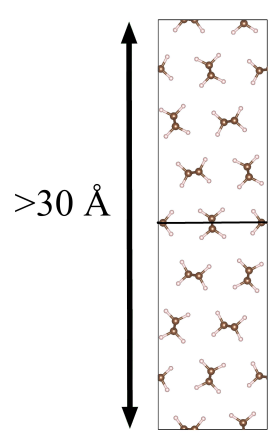

(a)

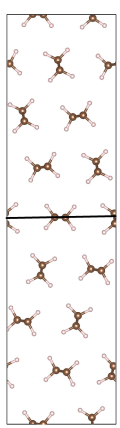

(b)

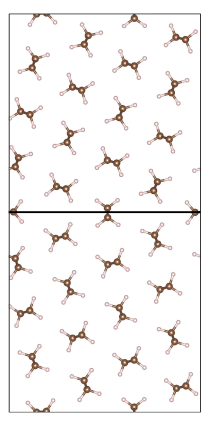

(c)

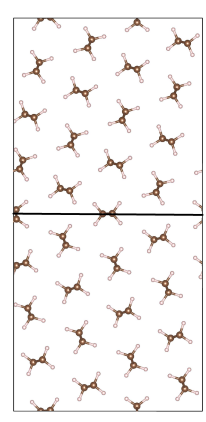

(d)
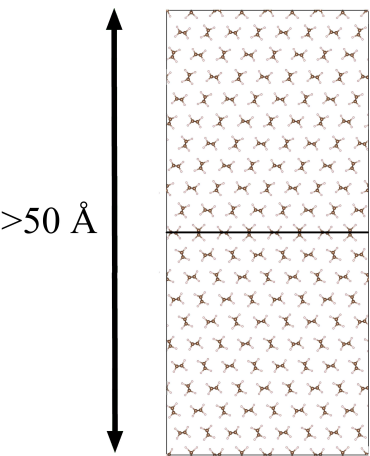

$>25 \AA$

(e)

Figure 2: Illustration of twin supercells utilized in the DFT modelling for the (a) mirror-symmetric $\{110\}$-twin (b) \{110\}-twin with the upper half sheared (1/2)[1 10$]$ relative to the lower half, (c) mirror-symmetric $\{310\}$-twin and (d) $\{310\}$-twin with the upper half sheared (1/2)[130] relative to the lower half. (e) Schematic supercell geometry for the twin modelling using classical simulations with the OPLS-AA interactions scheme. The horizontal lines indicate the twins in the center of the supercell, whereas the other interfaces are found at the supercell boundary.

we investigate those depicted in Figure 2(a) and (c), which correspond to perfect mirror-symmetric twins. But to explore the other possible energy minima, as illustrated in Figure 2(b) and (d), we also translate the upper and lower halves of the supercell by half of the translation vectors, i.e. (1/2)[1 $1 \overline{1} 0]$ and $(1 / 2)[1 \overline{3} 0]$ for the $\{110\}$ and $\{310\}$ twins, respectively. Moreover, although not depicted, we also consider shearing corresponding to a (1/2)[001] translation. All these combinations give a total of four twin configurations that are considered for each interface type. These different configurations are allowed to undergo atomic relaxations in all directions while simultaneously letting the supercell undergo stress relaxation in the outof-plane direction of the interface. This allows the supercell to vary in size to accommodate local expansion at the twin boundary, which emerges as a result of the non-ideal local atomic arrangement in its vicinity.

As depicted in Figure 2, each supercell contains two twin boundaries, which means that the interface energy can be calculated as

$$
\gamma_{T}=\frac{E_{T}-N E_{C H_{2}}}{2 A}
$$

where $E_{T}$ is the energy of the supercell containing the twins, $N$ is the number of $\mathrm{CH}_{2}$-units contained within the supercell, $\mathrm{E}_{\mathrm{CH}_{2}}$ is the bulk energy per $\mathrm{CH}_{2}$-unit in the orthorhombic phase and $A$ is the interface area. For reliable results of the twin boundary energy the two interfaces may not interact and it is therefore essential that they are well separated. This is particularly important for the DFT modelling, where there are also severe numerical constraints that limit the system size. To this end we chose the supercell dimension in the twin normal direction to be no less than $30 \AA$, which was found sufficient to obtain well-converged results, see Figure 2(a)-(d). Thus, the supercells depicted in Figure 2(a) and (b) contain $32 \mathrm{CH}_{2}$-units (i.e. 96 atoms) while those in (c) and (d) contain $64 \mathrm{CH}_{2}$-units (i.e. 192 atoms). For the classical atomistic modelling, the supercells are made sufficiently large that the minimum periodic im- age criterion is not violated. To comply with this requirement we chose the supercell dimension normal to the twin boundary to be at least $50 \AA$, and $25 \AA$ for the in-plane dimensions, see Figure 2(e).

\subsection{Generalized stacking fault energy}

To investigate the resistance to shearing and to gain insight on the stacking fault characteristics, we compute the $\gamma$-surfaces [69] for the three most frequently active slip planes observed experimentally: (100), (010) and (110), see Figure 3(a). This is carried out by gradually shearing the periodic supercells by imposing in-plane shearing displacements and mapping out the energy vs. displacement. For each shearing increment all carbon atoms in the supercell are allowed to relax only in the direction normal to the shearing plane, while no restriction is put on the coordinate relaxation of the $\mathrm{H}$-atoms. This modelling strategy ensures that the prescribed shearing is preserved.

To obtain well-converged results of the stacking fault energies, supercells with increasing dimensions perpendicular to the shearing plane were studied. For instance, for the (110)-system we found that supercells with a height of $25 \AA$ were of more than sufficient size to yield converged results. Hence, for all the computations of the unstable stacking fault energies the supercell dimensions perpendicular to the shearing plane were chosen to be no less than $25 \AA$ to ensure a reliable account of the $\gamma$-surface, cf. Figure 3(b)-(d).

\subsection{Numerical details}

\subsubsection{DFT modelling}

All DFT simulations in this work are performed using the open-source plane-wave based Quantum Espresso (QE) software suite [70]. For describing the electron-ion interaction we make use of the ultrasoft pseudopotential approach [71, 72] with the electron description for $\mathrm{C}$ comprising the $2 \mathrm{~s}^{2} 2 \mathrm{p}^{2}$ states, while that of $\mathrm{H}$ consists of a $1 \mathrm{~s}^{1}$ electron. 


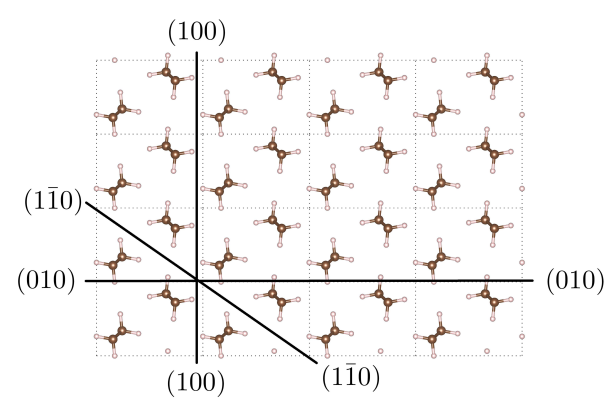

(a)

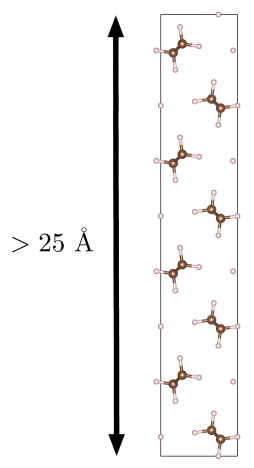

(b)

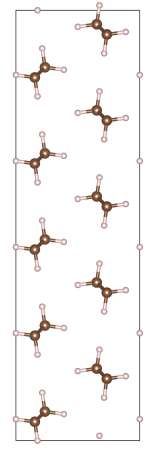

(c)

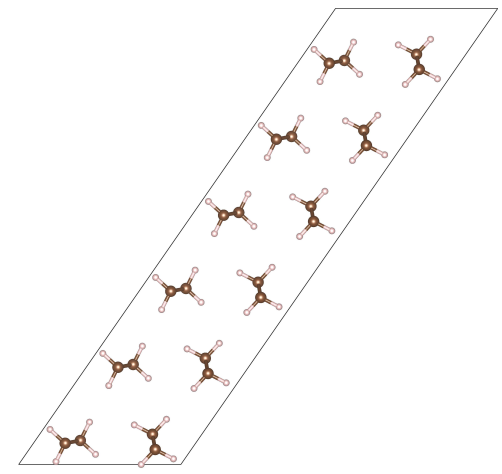

(d)

Figure 3: (a) Illustration of (100), (010) and (11̄0) slip planes. Schematic illustrations of the utilized supercells for modelling of slip on the (b) (100), (c) (010) and (d) (11̄0)-slip planes

We chose the kinetic energy cutoff for the plane-wave basis set and $k$-point density such that the ground state energy is converged within less than $0.5 \mathrm{meV} /$ atom. To achieve this, we have used a kinetic energy cutoff of $60 \mathrm{Ry}(1 \mathrm{Ry}=13.6 \mathrm{eV})$ and the energy cutoff for the electron density is set to 600 Ry. A $k$ point grid for the first Brillouin zone of the orthorhombic unit cell for PE corresponding to a $\Gamma$-centered $4 \times 4 \times 10$ sized grid generated by means of the Monkhorst-Pack method [73], was found sufficient to produce well converged results. The $k$-point mesh for the supercells used in the $\gamma$-surface and twin boundary simulations are made commensurate with the orthorhombic cell.

\subsubsection{Classical atomistic modelling}

For the classical atomistic modelling we use the LAMMPS software [74] and for the interatomic interaction, we adopt two existing strategies: the OPLS-AA and UA approaches. The first of these retains a fully atomistic representation, whereas the second provides a coarse-grained description where the hydrogen atoms are lumped together with the carbon to form united $\mathrm{CH}_{2}$ particles, with effective interactions described by an effective coarse grained force field. The parameters for the OPLSAA field are described in [75-77] and the UA-potential parameters originate from Paul et al. [78], but were subsequently modified by Bolton et al. [79] and by in't Veld and Rutledge [80]. For clarity and the purpose of later discussion we summarize the contributions to the potential energy for the two force fields here.

For the covalent bonds, the bond stretch, $E_{\text {bond }}$, and angle bendings, $E_{\text {angle }}$, are represented by harmonic potentials, i.e.

$$
E_{\text {bond }}=\frac{1}{2} K_{b}\left(r-r_{0}\right)^{2}
$$

and

$$
E_{\text {angle }}=\frac{1}{2} K_{\theta}\left(\theta-\theta_{0}\right)^{2}
$$

while the dihedral chain deformations are governed by a cosine series potential

$$
E_{\text {dihedral }}=\frac{1}{2} \sum_{n=1}^{3} K_{n}\left(1-(-1)^{n} \cos n \phi\right)
$$

The non-bonded interaction consists of vdW-interaction and repulsion described by the L-J 12-6 potential

$$
E_{v d W}=4 \epsilon\left[\left(\frac{\sigma}{r}\right)^{12}-\left(\frac{\sigma}{r}\right)^{6}\right]
$$

For the OPLS-AA potential there are partial charges assigned to the $\mathrm{C}$ and $\mathrm{H}$ atoms that give rise to Coulombic interaction, which is absent in the UA approach because of the neutral charging of the lumped $\mathrm{CH}_{2}$-particles. The partial charge for $\mathrm{H}$ atoms is +0.06 , while that of $\mathrm{C}$ is determined by charge neutrality. For the adopted potentials the L-J contributions are limited by a cutoff at $12 \AA$ for OPLS-AA and $10 \AA$ for UA, while the Coulomb interactions are unbounded and computed using the $k$-space pppm algorithm implemented in LAMMPS. The explicit parameters of the potentials can be seen in Table 1.

\section{Results and discussion}

\subsection{Lattice parameters and elastic response}

To validate our use of vdW-DF-cx, a nonlocal-correlation DFT, for this soft-matter problem, we compute the lattice parameters of the orthorhombic unit cell. In Table 2 we contrast the DFT results with those from experimental measurements and other, earlier DFT works, some of them with an earlier version of the vdW-DF method. Through relaxation of the unit cell we find that $c=2.55 \AA$, which concurs well with experimental data published in the literature [81, 82] cf. Table 2. This good agreement is expected in light of the fact that binding in the $c$ direction is primarily covalent, which can be routinely captured by most DFT formulations. 
Table 1: Parameters of the empirical potentials. $K_{\theta}, K_{n}$ and $\epsilon$ are given in $\mathrm{kcal} / \mathrm{mol}, K_{b}$ is given in $\mathrm{kcal} /\left(\mathrm{mol} \cdot \AA^{2}\right)$ and $r_{0}$ and $\sigma$ are given in $\AA$.

\begin{tabular}{lllll}
\hline OPLS-AA & & & & \\
\hline $\mathrm{C}-\mathrm{C}$ & $K_{b}=536$ & $r_{0}=1.529$ & $\epsilon=0.066$ & $\sigma=3.50$ \\
$\mathrm{C}-\mathrm{H}$ & $K_{b}=680$ & $r_{0}=1.090$ & $\epsilon=0.045$ & $\sigma=2.96$ \\
$\mathrm{H}-\mathrm{H}$ & & & $\epsilon=0.030$ & $\sigma=2.50$ \\
$\mathrm{C}-\mathrm{C}-\mathrm{C}$ & $K_{\theta}=118$ & $\theta_{0}=112.7^{\circ}$ & & \\
$\mathrm{C}-\mathrm{C}-\mathrm{H}$ & $K_{\theta}=75.0$ & $\theta_{0}=110.7^{\circ}$ & & \\
$\mathrm{H}-\mathrm{C}-\mathrm{H}$ & $K_{\theta}=66.0$ & $\theta_{0}=107.8^{\circ}$ & & \\
$\mathrm{C}-\mathrm{C}-\mathrm{C}-\mathrm{C}$ & $K_{1}=1.74$ & $K_{2}=-0.157$ & $K_{3}=0.279$ & \\
$\mathrm{C}-\mathrm{C}-\mathrm{C}-\mathrm{H}$ & $K_{1}=0.0$ & $K_{2}=0.0$ & $K_{3}=0.366$ & \\
$\mathrm{H}-\mathrm{C}-\mathrm{C}-\mathrm{H}$ & $K_{1}=0.0$ & $K_{2}=0.0$ & $K_{3}=0.318$ & \\
\hline $\mathrm{UA}$ & & & & \\
\hline $\mathrm{CH}_{2}-\mathrm{CH}_{2}$ & $K_{b}=634$ & $r_{0}=1.530$ & $\epsilon=0.093$ & $\sigma=4.009$ \\
$\mathrm{CH}_{2}-\mathrm{CH}_{2}-\mathrm{CH}_{2}$ & $K_{\theta}=120$ & $\theta_{0}=110^{\circ}$ & & \\
$\mathrm{CH}_{2}-\mathrm{CH}_{2}-\mathrm{CH}_{2}-\mathrm{CH}_{2}$ & $K_{1}=1.6$ & $K_{2}=-0.867$ & $K_{3}=3.24$ & \\
\hline
\end{tabular}

Unlike $c$, the lattice parameters $a$ and $b$ are highly dependent on the adopted exchange-correlation functional. This can be attributed to the fact the intermolecular interaction is governed by long range dispersive vdW forces, which in general are not captured by conventional dense-matter DFT, when using the generalized gradient approximation (GGA) or the local density approximation (LDA) for $E_{\mathrm{xc}}$.

Figure 4 reports our vdW-DF-cx characterization for the PE crystal binding energy variation with lattice parameters $a$ and $b$ (at the optimal $c$ values) and a comparison with data from both experiments and previous DFT studies. We note that the computed lattice parameters correspond to the ground state structure at $T=0 \mathrm{~K}$ (without corrections for zero-point vibrational effects) for a defect free crystal. In contrast, the experimental data are obtained for temperatures in the range 4-90 $\mathrm{K}$ for samples containing defects, and a direct comparison between DFT and experimental data can be somewhat misleading. Nevertheless, as summarized in Table 2, DFT using the vdW-DF-cx functional does quite accurately capture the experimentally observed results for $a$ and $b$. The experimental values of $a$ range from 7.12 to $7.42 \AA$, while $b$ spans from 4.85 to $4.96 \AA$. These values agree well with our equilibrium vdW-DF-cx values of

Table 2: Computed and experimentally measured lattice parameters. The data is presented in $\AA$.

\begin{tabular}{lccc}
\hline Source & $a$ & $b$ & $c$ \\
\hline DFT (vdW-DF-cx, this work) & 7.218 & 5.024 & 2.553 \\
DFT (vdW-DF, [22]) & 7.30 & 5.22 & 2.57 \\
DFT (LDA, [83]) & 6.549 & 4.446 & 2.515 \\
DFT (LDA, [84]) & 6.73 & 4.53 & 2.52 \\
DFT (PBE-GGA, [83]) & 7.304 & 5.017 & 2.565 \\
DFT (PBE-GGA, [84]) & 8.28 & 5.64 & 2.57 \\
MD (OPLS-AA, this work) & 7.284 & 4.908 & 2.541 \\
MD (UA, this work) & 7.748 & 4.463 & 2.518 \\
Exp (X-ray, 77 K [85]) & 7.42 & 4.96 & - \\
Exp (X-ray, 77 K [81]) & 7.388 & 4.929 & 2.539 \\
Exp (Neutron, 4 K [82]) & 7.121 & 4.851 & 2.548 \\
Exp (Neutron, 90 K [82]) & 7.161 & 4.866 & 2.546 \\
\hline
\end{tabular}

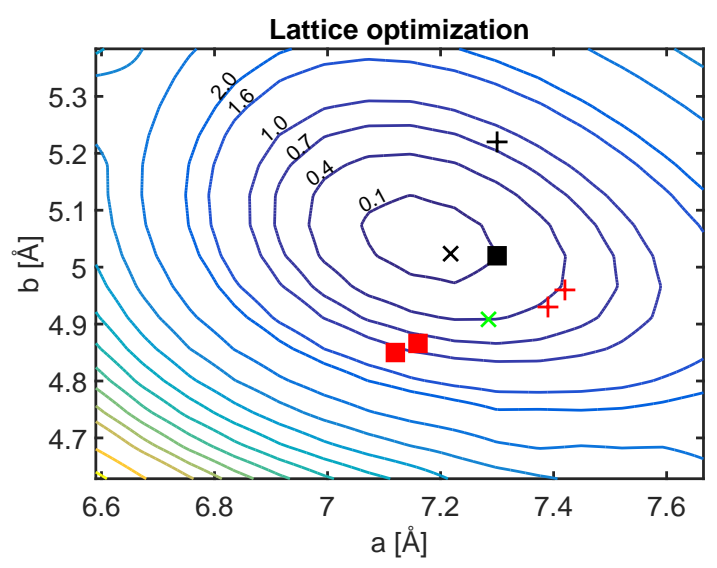

Figure 4: Energy contour plot for the orthorhombic unit cell. The indicated energy levels are measured in units of $\mathrm{meV} / \mathrm{CH}_{2}$. The black, red and green markers correspond to DFT, experimental and classical molecular dynamics data, respectively. The black and green $\mathrm{x}$-symbol corresponds to the optimized lattice parameters for the DFT and OPLS-AA approaches, while the black + and $\square$-markers correspond to DFT data from [22] and [83], respectively. The red + symbols represent $\mathrm{X}$-ray experiments performed at $77 \mathrm{~K}[81,85]$ and the red $\square$-markers represent results from neutron diffraction experiments performed at $4 \mathrm{~K}$ and $90 \mathrm{~K}$ [82].

7.22 and $5.02 \AA$, respectively. This good agreement with experimental data is in line with the results of Rangel et al. [60] and Brown-Altvater et al. [61], who demonstrated an excellent reliability of vdW-DF-cx for predicting the structure and vibrations of organic crystals and who documented its potential use as a starting point for also characterizing optical excitations.

From Table 2, it is seen that lattice parameters computed based on LDA are substantially underestimated, which is due to the well known overbinding $[86,87]$ and that the LDA has no account of vdW forces and gives no transferable account of soft matter [87]. PBE-GGA modelling is found to predict lattice parameters that are in quite good agreement with experimental data [83]. However, GGA-DFT lacks an account of vdW forces and it also suffers from other deficiencies such as a high stiffness related to normal loading in the $a$ and $b$ directions. Specifically in [83] it was reported that $C_{11}=39.2 \mathrm{GPa}$ for PBE- 
Table 3: Twin boundary energies, $\gamma_{T}$, for the different modelling strategies, given in $\mathrm{mJ} / \mathrm{m}^{2}$

\begin{tabular}{lccc}
\hline Twin type & DFT & OPLS-AA & UA \\
\hline$\{110\}$ mirror-symmetric & 8.9 & 29 & 28 \\
$\{110\}$ translated $(1 / 2)[1 \overline{1} 0]$ & 13 & 39 & 20 \\
$\{110\}$ translated $(1 / 2)[001]$ & 15 & 57 & 20 \\
$\{110\}$ translated $(1 / 2)[1 \overline{1} 1]$ & 17 & 33 & 20 \\
$\{310\}$ mirror-symmetric & 28 & 38 & 19 \\
$\{310\}$ translated $(1 / 2)[1 \overline{3} 0]$ & 46 & 45 & 17 \\
$\{310\}$ translated $(1 / 2)[001]$ & 36 & 78 & 18 \\
$\{310\}$ translated $(1 / 2)[1 \overline{3} 1]$ & 44 & 65 & 18 \\
\hline
\end{tabular}

GGA calculations, while the available experimental data lie in the range 8.4-11.5 $\mathrm{GPa}[88,89]$. The transverse bonding in $\mathrm{PE}$ is simply not accurately represented by PBE-GGA calculations. In the present vdW-DF-cx work we computed $C_{11}=8.8$ $\mathrm{GPa}$, which agrees well with the experimental data. We note that vdW-DF-cx is free of all parameter input and that our calculations effectively predict the materials behavior without any parameter input. In light of the good predictive capabilities, reported above, we are confident that this nonlocal-correlation DFT is suitable also for the broader exploration that is included in the present work.

The performance of the adopted empirical potentials for classical MD is also evaluated and reported in Table 2. It is seen that the $c$-parameter concurs well with experimental data for both the OPLS-AA and UA approaches. As shown in Figure 4, the OPLS-AA potential accurately does predict the $a$ and $b$ lattice parameters. The respective values of 7.28 and $4.91 \AA$ fall within the aforementioned range of experimental data. However, the UA data predict $a=7.75$ and $b=4.46 \AA$, which deviate by up to $10 \%$ from the experimental data.

\subsection{Twin boundaries}

Twinning is the second-most commonly occurring plastic mechanism in crystalline PE. The more prominent slip processes are discussed below. However, twin boundaries are closely linked to the questions of stability and ground-state (or metastable-state) configurations, and hence are a natural stepping stone for our broader exploration.

We consider the most frequently occurring twin boundaries, $\{110\}$ and $\{310\}$ twins, and compare the energy differences among variants. Specifically, using our nonlocal DFT, and our pair of classical MD simulations we compute the optimal structure and total formation energy for each such twin candidate.

Table 3 reports and compares these twin boundary energies, $\gamma_{T}$. The grain boundary energies for the $\{110\}$-twins computed by means of DFT span from 9 to $17 \mathrm{~mJ} / \mathrm{m}^{2}$, while the OPLS-AA energies range from 29 to $57 \mathrm{~mJ} / \mathrm{m}^{2}$. For the $\{310\}$-twins they span from 28 to $46 \mathrm{~mJ} / \mathrm{m}^{2}$ and 38 to $78 \mathrm{~mJ} / \mathrm{m}^{2}$, respectively. Although the energy differences are not large, the results imply that the mirror symmetric twin boundaries are most stable. Also, the all-atom methods (DFT and OPLS-AA) both consistently find the grain boundary energy for $\{110\}$-twins are clearly lower than those of $\{310\}$-twins. In contrast, the UA model essentially finds that all twin configurations have energies of the same order, $\sim 20 \mathrm{~mJ} / \mathrm{m}^{2}$. The fact that the UA-model fails to differentiate the grain boundary energies of the different types and shifts suggests that an all-atom (or electron-based, DFT) description is essential for an accurate prediction of the ground state of twin boundaries.

A similar MD investigation by Geary and Bacon [19] predicted that the $\{110\}$-twin boundary energies will lie in the range $11-14 \mathrm{~mJ} / \mathrm{m}^{2}$. Geary and Bacon used a simple hybrid Buckingham and Born-Mayer pair potential in conjunction with a rigid molecule assumption to describe the interatomic interaction. Their twin-boundary energy value corresponds well with our $a b$ initio DFT results. They furthermore found the energies for $\{310\}$-twins to lie in the range $32-52 \mathrm{~mJ} / \mathrm{m}^{2}$, which also agrees well with both our DFT and OPLS-AA results.

The surprisingly good agreement between Ref. [19] and our DFT and OPLS-AA results can be partially attributed to the emphasis on describing the $\mathrm{vdW}$ interactions. The attractive part of the Buckingham potential contains a $r^{-6}$ dependence and thus describes the averaged interaction between temporary dipoles responsible for the $\mathrm{vdW}$-interaction. This suggests that the relatively simple approach may give a good representation of metastable-states. However, there are also important differences. For example, Geary and Bacon [19] predict that the (1/2)[001] and (1/2)[131] shifted grain boundaries should have the lowest grain boundary energy. In contrast, our results imply that the mirror-symmetric twins are most stable, cf. Table 3.

\subsection{Generalized stacking fault energy}

To investigate the resistance to shearing in crystalline PE, we have computed the $\gamma$-surfaces for slip on the (100), (010) and (110)-planes. These are obtained by incrementally imposing shear deformations on the supercell vectors, such that a slip interface is obtained between the neighbouring supercells, and mapping the energy vs. displacement. The optimal shearing path is identified as that having the lowest energy threshold.

\subsection{1. (100)-slip plane}

Figure 5(a)-(c) show contour plots of the $\gamma$-surface for the (100)-slip plane for DFT, OPLS-AA and UA, respectively. The computed energy surfaces indicate a maximum threshold for shearing occurring for the displacement (1/2)[011]. The magnitude of this maximum value depends on the modelling approach, but is found to correspond to $\sim 150 \mathrm{~mJ} / \mathrm{m}^{2}$ for the DFT modelling. As illustrated in Figure 5(b)-(c), the classical atomistic modelling schemes predict lower thresholds than found for DFT. Specifically, they lie in the range $40-60 \mathrm{~mJ} / \mathrm{m}^{2}$.

From Figure 5(a)-(c), it is found that the path of least resistance occurs for [001]-slip for all force fields. Figure 5(d) reports that the observed energy thresholds correspond to 12 $\mathrm{mJ} / \mathrm{m}^{2}$ for DFT and $9 \mathrm{~mJ} / \mathrm{m}^{2}$ for OPLS-AA, while that for the UA-force field is only $3 \mathrm{~mJ} / \mathrm{m}^{2}$. This mode represents chain slip, which because of the low activation energy is the most likely slip mechanism to be active, in accordance with experimental observations $[4-6,9,11]$. The fact that the iso-lines in 

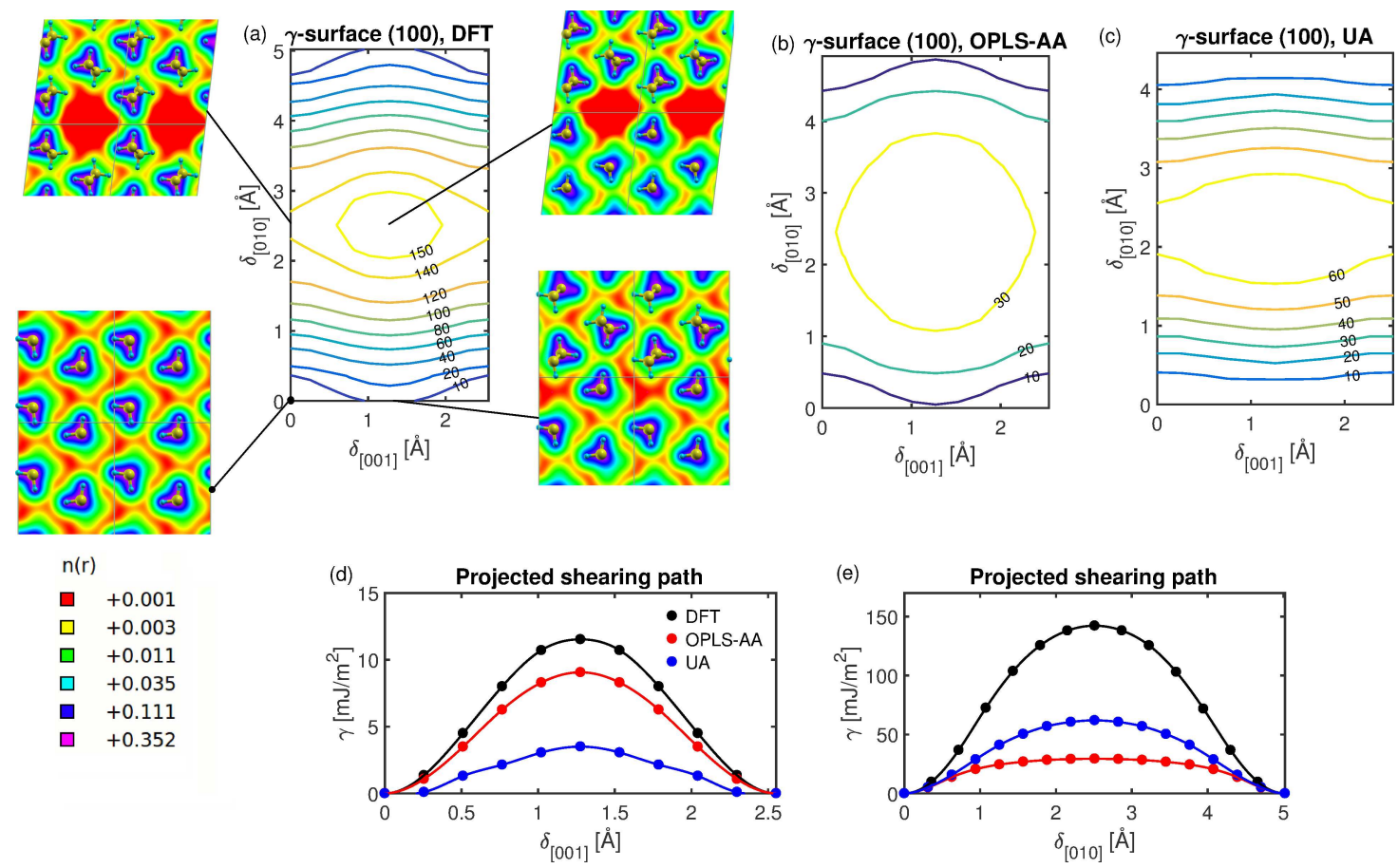

Figure 5: The $\gamma$-surface for shearing of the (100)-plane for (a) DFT, (b) OPLS-AA and (c) UA force fields. The data in (a)-(c) are given in units of mJ/m². The insets in (a) correspond to the logarithmic charge density plots around the slip plane projected in the [100]-direction for different amounts of displacements generated using the xCrysDen software [90]. The lower panels show the projected curves along the (d) [001] and (e) [010]-directions. The markers correspond to computed data points coinciding with the paths, whereas the solid lines correspond to interpolated curves extracted from the $\gamma$-surface using cubic splines.

Figure 5(c) are more or less horizontal flags for a shortcoming of the UA potential in predicting chain slip. The lack of amplitude variation yields low threshold energies, which can be derived from the lack of explicit close ranged repulsive $\mathrm{H}-\mathrm{H}$ interaction. We shall return to this point in a discussion of the nature of the barrier for slip dynamics, below.

The path for transverse slip that displays the lowest energy threshold can be identified in Figure 5(a)-(c) as that for [010]slip. The energy curve for this projected slip path is seen in Figure 5(e), from which it is deduced that the DFT modelling predicts a maximum energy of $\sim 140 \mathrm{~mJ} / \mathrm{m}^{2}$, which is of the order of ten times higher than that of the chain slip. The threshold energies for OPLS-AA and UA force fields are substantially lower than the DFT data, corresponding to about 30 and 60 $\mathrm{mJ} / \mathrm{m}^{2}$, respectively.

\subsection{2. (010)-slip plane}

The $\gamma$-surfaces for (010)-slip are shown in Figure 6(a)-(c). The energy curves for the chain slip mechanism for the different force fields are depicted in Figure 6(d), and the energy threshold obtained from DFT-modelling is $\sim 17 \mathrm{~mJ} / \mathrm{m}^{2}$. This is of the same order as for chain slip on the (100) slip plane and it compares well with the OPLS-AA result of $15 \mathrm{~mJ} / \mathrm{m}^{2}$, while that for the UA force field, similar to the (100)-slip plane, is substantially lower, $\sim 3 \mathrm{~mJ} / \mathrm{m}^{2}$. The DFT curve also reveals that there is a local minimum for the (1/2)[001] slip vector. However, the depth of this minimum is only of the order of $3 \mathrm{~mJ} / \mathrm{m}^{2}$, cf. Figure 6(d), which suggests that it will have no practical impact on slip. This shallow minimum is likely a symmetry effect that will disappear when thermal vibrations are accounted for.

By comparing the energy levels with that of the (100) $\gamma$ surface, it is seen that the threshold energy for transverse slip in general is higher for the (010)-slip plane. This is particularly true for pure transverse slip along the [100]-direction for which the threshold energy is almost twice that for (100)[010]-slip, see Figure 6(e). This generally higher energy level related to transverse slip is in line with the fact that the [100]-Burgers vector is longer than the [010]-Burgers vector, in agreement with also experimental observations of higher resistance to such slip [3]. Again, we observe that the DFT and UA force fields have similar thresholds for transverse slip, $\sim 230-280 \mathrm{~mJ} / \mathrm{m}^{2}$, while the slip threshold for the OPLS-AA parameterization is about one third of those.

The $\gamma$-surfaces reveal that there is an unstable state, i.e. a maximum, for the slip vector corresponding to $(1 / 2)[100]$, while there is a saddle point of lower energy for the (1/2)[101] slip vector. To investigate this saddle point closer in Figure 6(f) we have plotted the slip energy for an alternative composite slip path that consists of three sequential parts $((1 / 2)[001]+[100]+(1 / 2)[00 \overline{1}])$, as depicted in Figure 6(a)-(c). The energy threshold is only slightly lower $\left(\sim 220-260 \mathrm{~mJ} / \mathrm{m}^{2}\right.$ for DFT and UA and $\sim 70 \mathrm{~mJ} / \mathrm{m}^{2}$ for OPLS-AA) for this path. This indicates that transverse slip may be accompanied by a component of chain slip to reduce the activation energy.

\subsection{3. (1 $1 \overline{1} 0)$-slip plane}

The final $\gamma$-surfaces considered are those for the (110)-slip plane, depicted in Figure 7(a)-(c). The first observation is that 

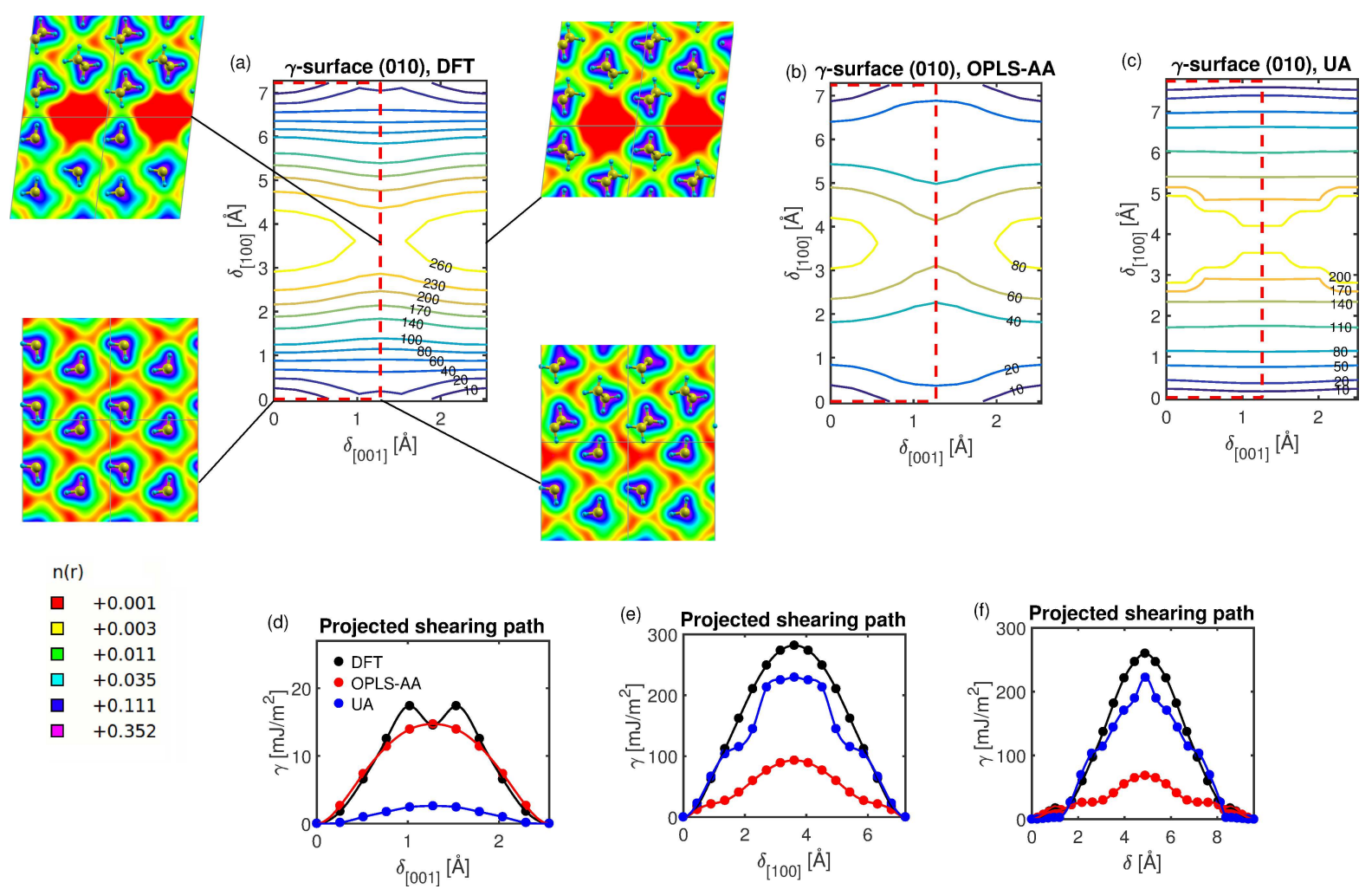

Figure 6: The $\gamma$-surface for shearing of the (010)-plane for (a) DFT, (b) OPLS-AA and (c) UA force fields. The data in (a)-(c) are given in units of mJ $/ \mathrm{m}^{2}$ and the dashed red line corresponds to the studied path in (f). The insets in (a) correspond to the logarithmic charge density plots around the slip plane projected in the [010]-direction for different amounts of displacements. The lower panels show the projected curves along the (d) [001], (e) [100]-directions and (f) those for the paths illustrated in (a)-(c) by red dashed lines. The markers in (d)-(f) correspond to computed data points coinciding with the paths, whereas the solid lines correspond to interpolated curves extracted from the $\gamma$-surface using cubic splines.

the energy levels for the slip generally are lower than those of the (100) and (010) slip planes. This can be attributed to the fact that the (110)-plane is the most densely packed plane of those considered, as further discussed in the following section.

For the pure chain slip, i.e. [001]-slip, the activation energies of the DFT and OPLS-AA force fields are comparable to those of the (100) and (010) slip planes. The activation energy is found to be about $16 \mathrm{~mJ} / \mathrm{m}^{2}$, see Figure 7(d), while that for UA is about $3 \mathrm{~mJ} / \mathrm{m}^{2}$. By adding a small transverse slip component to the chain slip, such that the slip is characterized by $[001] \rightarrow(1 / 2)[\kappa \kappa 1]+(1 / 2)[\bar{\kappa} \bar{K} 1]$ with $\kappa \approx 0.1$, the DFT modelling predicts a threshold energy that is slightly reduced, at 10 $\mathrm{mJ} / \mathrm{m}^{2}$, as shown in Fig. 7(a). The same observation can also be made for the OPLS-AA force field but then using $\kappa \approx 0.5$. Coarse-grained models (such as the UA potential) cannot be expected to fully reproduce the intrinsic characteristics of the $\gamma$-surface and we do not see this local minimum when using the UA approach. For pure transverse slip in the [110]-direction, the threshold energy is about $40 \mathrm{~mJ} / \mathrm{m}^{2}$ for DFT and UA, as shown in Figure 7(e), which is much less than observed for the previous slip planes.

By inspection of the computed $\gamma$-surfaces in Figure 7(a)-(c), for all force fields it is seen that they are non-convex with a shallow recess that contains two local minima situated at the sites corresponding to the slip vectors (1/2)[110] and (1/2)[111]. This is in accordance with predictions from empirical rigid molecular modelling [19] and it is an indication that dissociation of dislocations may occur into partial dislocations through the possible reactions

$$
[110] \rightarrow \frac{1}{2}[110]+\frac{1}{2}[110]
$$

and

$$
[110] \rightarrow \frac{1}{2}[111]+\frac{1}{2}[11 \overline{1}] .
$$

In turn, this implies that the transverse slip may occur through a mixed mode of transverse and chain slip. This could explain why transverse slip on the (110)-plane has been observed experimentally in the polar regions of the spherulite [8], in spite of the large Burgers vector of associated with the perfect dislocation.

From an energy perspective the stacking faults are similar, with a slight preference for the stacking fault energy corresponding to the (1/2)[111] displacement. The DFT study reveals that the stacking fault energy corresponds to $\sim 17 \mathrm{~mJ} / \mathrm{m}^{2}$, while they are calculated to be less than $10 \mathrm{~mJ} / \mathrm{m}^{2}$ for the OPLS-AA and UA force fields.

The transverse slip paths depicted by red dashed lines in Figure 7(a)-(c) correspond to the dissociation of (8) into smaller segments, which are found to follow low energy troughs and therefore pose as candidates for transverse slip. The energy curve corresponding to this slip movement is shown in Figure 

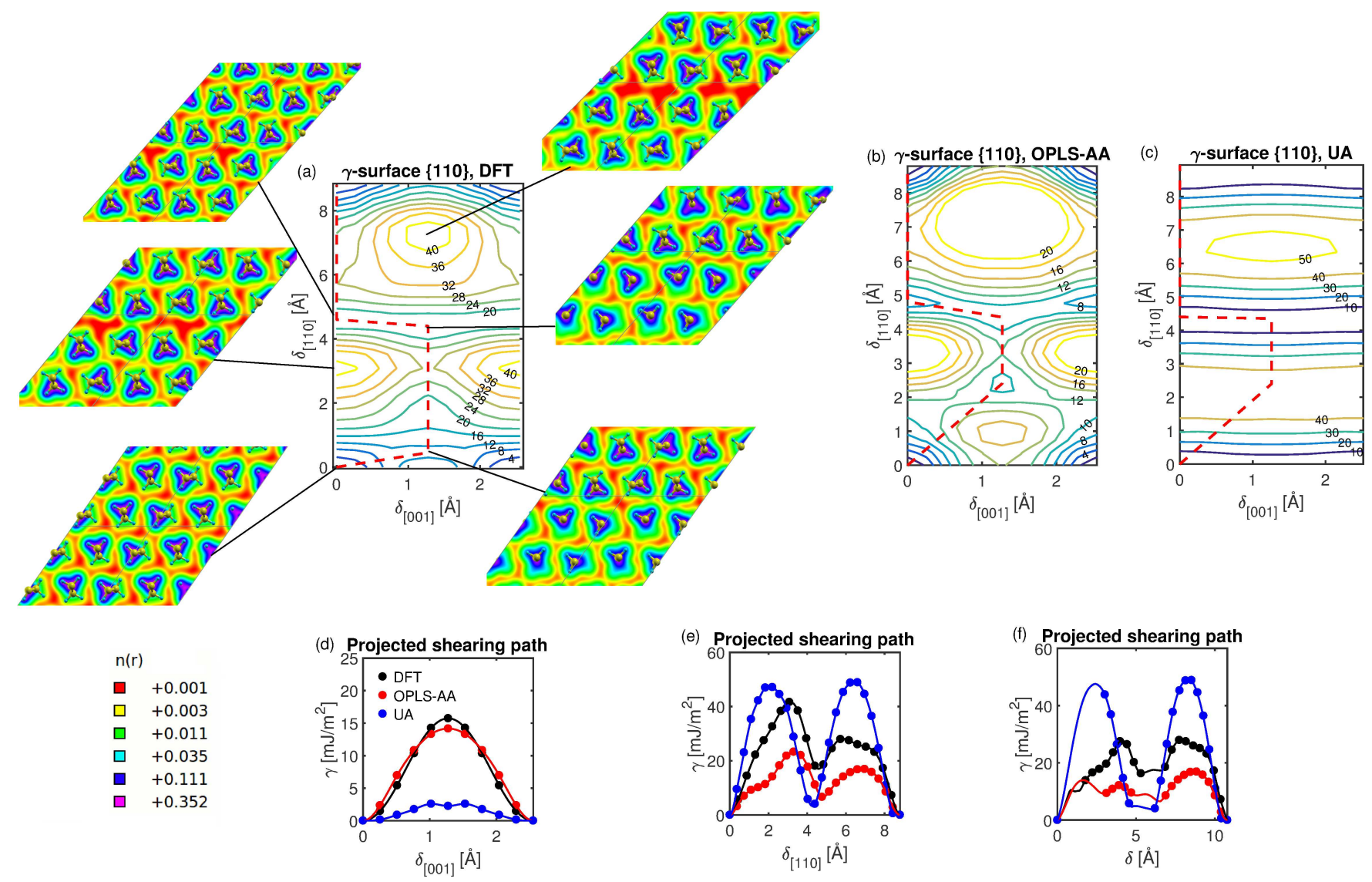

Figure 7: The $\gamma$-surface for shearing of the (110)-plane for (a) DFT, (b) OPLS-AA and (c) UA force fields. The data in (a)-(c) are given in units of mJ/m² and the dashed red line corresponds to the studied path in (f). The insets in (a) correspond to the logarithmic charge density plots around the slip plane projected in the [110]-direction for different amounts of displacements. The projected curves along the (d) [001], (e) [110]-directions and (f) those for the paths illustrated in (a)-(c) by red dashed lines. The markers in (d)-(f) correspond to computed data points coinciding with the paths, whereas the solid lines correspond to interpolated curves extracted from the $\gamma$-surface using cubic splines.

7(f), which reveals that the activation energy for this path is lower than that for pure transverse slip, cf. Figure 7(e).

\section{Nature of the slip-activation barriers}

Summarizing the surveys of the slip-activation barriers, above, we find that all modelling approaches predict a preference towards chain slip, in accordance with experimental observations. However, while the activation energies for such slip obtained from DFT and OPLS-AA modelling are similar, that obtained using UA is substantially lower. Moreover, the observed variation of the slip energy landscape is for all practical purposes negligible for chain slip of the UA potential. In fact, the iso-lines in Figures 5(c), 6(c) and 7(c) are all more or less horizontal.

The slip processes are governed by the competition between vdW attraction and Pauli-exclusion between neighboring groups, because such deformations do not include any notable molecular stretching, bond bending or rotation. The UA model is coarse grained, treating each $\mathrm{CH}_{2}$ group as one particle and the intermolecular $\mathrm{CH}_{2}-\mathrm{CH}_{2}$ coupling by just a single per-group effective L-J potential. The generic, per-atom, L-J descriptions has both an attractive part (reflecting vdW forces) and a repulsive component (reflecting steric hindrance effects). DFT, using vdW-DF-cx, can quantify the magnitude of such components, making clear that the Pauli exclusion actually has an exponential dependence on separations; It is by far the most rapidly varying component and it depends critically on the atomic configuration. The UA L-J potential part should be seen as a weighted average of underlying attractive and repulsive components-but one cannot expect that the same average can be used for all interface geometries that define a slip process.

Specifically, we ascribe the UA failure to reproduce the chain slip energy, Figs. 5(d), 6(d) and 7(d), to the fact that it does not have a transferable account of the role played by the strong $\mathrm{H}-\mathrm{H}$ interactions. The hydrogen atoms stick out of the $\mathrm{CH}_{2}$ groups but in the ground state configuration there is a mutual alignment, thus minimizing the Pauli exclusion term. However, when the slip process causes two hydrogens to pass each other, the steric hindrance causes a velcro-type blockade under which the UA would need a different per-group, effective L-J parameterization. The UA fails because it is not properly set up to track the changes that arises in the mutual $\mathrm{H}-\mathrm{H}$ repulsion under such conditions.

To gain further insight on the physics behind the slip energy 
landscape, detailing the above-stated $\mathrm{H}$-atom velcro picture, we investigate the charge density distribution around the set of investigated slip planes. The density-variation plots, shown as inserts of Figs. 5(a), 6(a), and 7(a), are evaluated at the extreme or barrier points in the energy landscape, identified by arrows.

We begin with a characterization of the (100)-slip plane. The charge density plots for the (100)-slip plane, in Fig. 5(a), reveal that any displacements including a transverse slip component leads to the formation of both (i) regions of exceptionally low electron density in the slip plane, and (ii) regions (between $\mathrm{H}$ atoms on neighboring molecules) with high-electron concentration. The formation of '(i)'-low-density and '(ii)'high-density inter-hydrogen regions are observed whenever the $\mathrm{H}-\mathrm{H}$ separations are small, conditions that correlate with high threshold energies for slip. Because the (100) slip plane is not densely packed, and as the transverse slip occurs along the [010]-direction, the molecule chains above and below the slip plane repel each other at the barrier configuration. In turn, this repelling causes the observed formations of regions '(i)' and '(ii)'. The effects are most significant when the transverse displacement component is at (1/2)[010], cf. Figure 5(a).

The charge density plots for the (010)-slip plane, in Fig. 6(a), reveal similar behavior to those of the (100)-slip plane. Again at the maxima and saddle points associated with transverse slip, the transverse displacements along the [100]-direction yield large regions of low charge density as well as regions with unusually large electron concentrations between two hydrogens on neighboring atoms. On the other hand, the charge density plots for the (110)-slip plane, in Fig. 7(a), reveal relatively small regions of depleted charge density and less pronounced density pockets between the pair of hydrogens. This slip plane is densely packed and thus more molecules are in proximity. More modest electron-density changes are still found in the vicinity of the slip plane that correlate with low threshold energies associated with transverse slip of the (110)-slip plane. However, here the slip is not putting as large electron density concentrations between neighboring hydrogens.

With the formation of low-density regions '(i)' one would obtain no binding energy contribution from a traditional semilocal functional [21, 23, 30, 87], but the same is not true for our nonlocal DFT, using vdW-DF-cx [27, 31]. The vdW-DF methods provides cohesion also when there are important regions that have a sparse electron distribution [21, 28, 30] and that holds true also when defects enhance the internal spacings [63]. Our vdW-DF-cx calculations provide a realistic account of the cohesion at the slip interfaces (at barrier conditions) because it correctly describes the $\mathrm{vdW}$ forces as arising from a nearfield electrodynamics coupling of the electron-gas response - a mechanism that also describes binding among completely separate fragments $[28,30,59]$. On the other hand, the formation of such very-low-density regions also means an energy increase (effectively contributing to the slip-activation energy barrier), because the electron distribution is then further from the optical electron conditions for nonlocal-correlation binding, as discussed in Refs. [28, 43, 87].

At the same time, at such barrier conditions we find that the residual nonlocal-correlation binding in vdW-DF-cx must be offset exclusive by the Pauli repulsion at a set of few $\mathrm{H}$ $\mathrm{H}$ contact points; The set of inserts in Figs. 5(a)-7(a) shows that at barrier conditions, there are clear enhancements in the electron density in the $\mathrm{H}-\mathrm{H}$ regions, (as compared to the stable configurations). As the $\mathrm{H}$ atoms are already bound to the carbons in the $\mathrm{CH}_{2}$ groups these density changes are not associated with any binding. Instead, the density concentrations produce an enhanced Pauli exclusion: the $\mathrm{H}-\mathrm{H}$ regions effectively serve as stiff brushes that tend to block mutual sliding, precisely as a velcro system does. This is a second (we expect dominant) contribution to the slip activation barrier.

We test this barrier interpretation by using the flexibility of the all-atom classical MD with OPLS-AA potentials, selectively turning off various components of the intermolecular L-J contributions. In effect, we repeat the characterization of the slip energy landscape but by enabling or disabling various contributions we isolate and, as it turn out, highlight the key role played by the $\mathrm{H}-\mathrm{H}$ repulsion.

Figure 8 reports this analysis of the $\gamma$-surface of the (110)slip plane using the OPLS-AA potential with suitable modifications, as indicated. In Figs. 8(a) and (b) we have removed the intermolecular $\mathrm{C}-\mathrm{C}$ interaction and the $\mathrm{C}-\mathrm{C}$ and $\mathrm{C}-\mathrm{H}$ interactions, respectively. Contrasting these results we see the main characteristics of the $\gamma$-surface in Fig. 7(b) are retained and hence conclude that the L-J- components are not dominant in setting the slip barrier. We conclude that, as expected from the analysis of the electron-density variation, it must instead be the close ranged $\mathrm{H}-\mathrm{H}$ interaction that plays a central role in describing the slip properly.

The interpretation is further verified in Figs. 8(c) and (d), in which the $\mathrm{H}-\mathrm{H}$ interaction and $\mathrm{H}-\mathrm{H}$ and $\mathrm{C}-\mathrm{H}$ interactions have been omitted, respectively. Here it is seen that the associated energy landscapes depicted differ substantially from that in Fig. 7(b). Specifically, it can be seen that the troughs and recesses are not as deep as in Figs. 8(a) and (b). The fact that the isolines are more or less horizontal illustrates the importance of the $\mathrm{H}-\mathrm{H}$ interaction for crystal plasticity modelling. The consequence of this shortcoming is that the required critically resolved shear stress becomes underestimated. We conclude that methods which, like the UA potential, lack an explicit focus on describing the important $\mathrm{H}-\mathrm{H}$ repulsion, may lead to an exaggerated and non-physical occurrence of fine or coarse chain slips.

Finally, combining the density-variation analysis (from nonlocal DFT) and the selective-component analysis (via OPLSAA potential approach) allows us to better understand the differences between the two investigated classical MD approaches. We have already discussed why the OPLS-AA holds advantages over the UA for the description of the set of slip processes themselves. However, it is interesting that the UA seems to outperform the OPLS-AA potential for the transverse-slip characterization (even if that method does not have the advantages of a per-atom focus, let alone the nonlocal DFT advantages of having a direct electron-density focus [46]).

The charge-density plots in Figs. 5(a), 6(a) and 7(a), show that transverse slip properties depend heavily on the relatively strong monomer repulsion characteristics. The fact that the L-J 

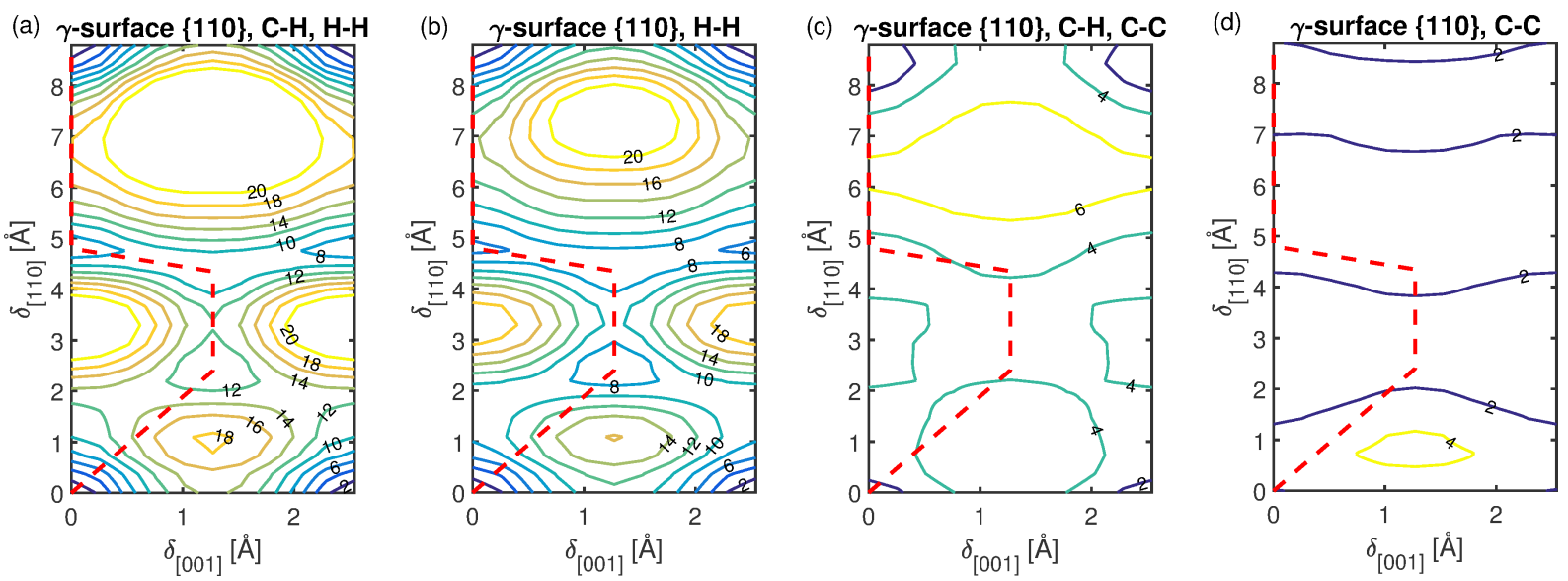

Figure 8: Illustration of different L-J contributions to the $\gamma$-surface for shearing of the (11̄0)-plane for the OPLS-AA potential. The energy contributions corresponding to the L-J potentials describing (a) the C-C, (b) the C-H and C-C, (c) the $\mathrm{H}-\mathrm{H}$ and (d) the C-H and $\mathrm{H}-\mathrm{H}$ interaction have been removed from the total energy to study their individual contributions.

component of the UA potential has relatively large $\sigma$ and $\epsilon$ (see Table 1), means that repulsion is activated at relatively large separations and becomes strong when the monomers approach each other. This is necessary as the UA works with effective L-J coefficients that are centered at the $\mathrm{CH}_{2}$ groups and not at the actual $\mathrm{H}$ positions. Nevertheless, it is still so that the UA approach yields transverse slip energy amplitudes, Figs. 5(c), 6(c) and 7(c), that concur fairly well with DFT data.

In contrast to the UA-potential approach, the L-J parts of the OPLS-AA potential are short-ranged (see Table 1) while at the same time the partial charges of $\mathrm{C}$ and $\mathrm{H}$ are low, -0.12 and +0.06 , respectively. Combined, these contributions to the OPLS-AA potential yield a weak monomer repulsion, which is why the transverse slip energy amplitude is low compared to the DFT data.

\section{Summary and conclusions}

In this work we have investigated the slip mechanisms and ground state twin configurations in crystalline orthorhombic polyethylene by means of $a b$ initio, or first principles, theory and classical atomistic simulations. The DFT modelling is performed using the recent consistent-exchange vdW-DF-cx version [27, 31] of the vdW-DF method, while the classical MD atomistic modelling is conducted using an all atom OPLS potential and a coarse-grained UA force field. Besides seeking an accurate characterization of the PE crystal defects and plasticity mechanisms, per se, we have also used the results of the strictly parameter-free vdW-DF-cx to assess and discuss broad applicability of the utilized classical MD modelling approaches.

We show that the vdW-DF-cx description accurately characterizes the ground state structure and elastic response of the PE crystal, and hence conclude that it serves as an accurate predictor of structure, cohesion and sliding forces in the related PE-defect problems [91]. As such it can be trusted to provide a detailed assessment of also the classical MD simulation approaches.
We have also studied the stability of twin boundaries in crystalline PE, contrasting our nonlocal DFT results and those of classical MD modeling using the OPLS-AA and OA potentials. We find that DFT and OPLS-AA yield results consistent with experiments. Specifically, we find that the $\{110\}$ type of twin boundary generally is characterized by a lower stacking fault energy than for the $\{310\}$ type. The mirror-symmetric twin boundaries have been found to have the lowest ground state energies. Typically, the twin boundary energies lie in the range $10-60 \mathrm{~mJ} / \mathrm{m}^{2}$ for the $\{110\}$-twins and $30-80 \mathrm{~mJ} / \mathrm{m}^{2}$ for the $\{310\}-$ twins.

For all considered slip planes it is found that there is a clear preference towards chain slip, which is in accordance with experimental observations. Typically the activation energies for such slip are of the order $10-20 \mathrm{~mJ} / \mathrm{m}^{2}$ as predicted by the DFT and OPLS-AA force fields, while it is found to be less than 5 $\mathrm{mJ} / \mathrm{m}^{2}$ for the UA potential.

This UA discrepancy is related to the lack of repulsion between adjacent monomer groups. The UA potential does not contain explicit $\mathrm{H}-\mathrm{H}$ repulsion, which becomes substantial when the interatomic distance is small. The absence of explicit $\mathrm{H}-\mathrm{H}$ repulsion leads to reduced chain slip energies, which may result in unphysical and excessive slip if it is utilized for modelling purposes.

For transverse slip of the (100) and (010), the DFT modelling predicts the threshold energies corresponding to 150 and $280 \mathrm{~mJ} / \mathrm{m}^{2}$, respectively. The results from the empirical potentials are found to be smaller. This is particularly evident for the OPLS-AA force field for which the activation energy is less than half of that predicted by our nonlocal DFT. Because transverse slip is characterized by very close ranged atomic interaction, this is again believed to be an artefact of weak monomer repulsion.

The $\gamma$-surfaces for the (110)-slip plane are found to be nonconvex with multiple potential energy minima, indicating the presence of stable stacking faults and the dissociation of perfect dislocations into partials. Especially two possible dislocation dissociation mechanisms have been identified. This is in 
line with the experimental observation of transverse slip on the (110)-slip plane in the polar regions of spherulites, which due to the large magnitude of the perfect dislocation otherwise would be unlikely [8]. The stacking fault energies for these stacking faults computed by means of DFT are found to be $\sim 17 \mathrm{~mJ} / \mathrm{m}^{2}$, while they are less than $10 \mathrm{~mJ} / \mathrm{m}^{2}$ for the UA and OPLS-AA potentials.

Overall, our results show that to capture the general characteristics of the $\gamma$-surface, it is necessary to utilize an all-atom model such as the OPLS-AA or a nonlocal DFT, such as vdWDF-cx. Although the amplitude of the $\gamma$-surface does not directly transfer to DFT data, much of the slip characteristics are captured by the relatively simple empirical potential and it will give a qualitative description of the slip properties. The shortcomings are rooted in the pre-specified interaction-potential parameters of empirical force fields, which limit the transferability. Nevertheless, the all-atom MD does permits us to use empirical input and still secure a qualitative description of defects and slip in the PE crystal.

Finally, by combining the predictability of nonlocal DFT and the flexibility of all-atom MD, we identify the nature of the slip activation energy barriers as caused by a steric hindrance between hydrogens on neighboring molecule. There is an enhanced Pauli exclusion repulsion as these $\mathrm{H}$ atoms are dragged past each other in the slip. We note that the here-discussed mechanisms for a sliding barrier is generic and that our findings may therefore also impact our understanding and modelling of general polymer and macromolecular systems.

On the one hand, our finding that attention to details of the Pauli exclusion is essential (for sliding and slip) sheds further light on the limitations of classical MD simulations: they lack the transferability and electron-density focus that nonlocal DFT naturally provides. On the other hand, this insight may also help us to a better use of classical MD modeling for important qualitative explorations. Depending on the property we wish to explore, the insight may, for example, guide us to pick a proper type of classical MD (all-atom versus coarse-grained) and seek to the proper empirical—or, when necessary, DFTbased-input on MD modelling parameters.

\section{Acknowledgments}

This work was funded by the Swedish Knowledge Foundation through grant no. 20150165, by the Swedish Research Council (VR), and by the Swedish Foundation for Strategic Research (SSF) under contract No. SE13-0061. The simulations were performed using computational resources provided by the Swedish National Infrastructure for Computing (SNIC) at the National Supercomputer Centre (NSC), Linköping University and at the High Performance Computing Center North (HPC2N), Umeå University.

\section{References}

[1] S. S. Katti, M. Schultz, The microstructure of injection-molded semicrystalline polymers: A review, Polym. Engng. Sci. 22 (16) (1982) 10011017.
[2] L. Lin, A. S. Argon, Structure and plastic deformation of polyethylene, J. Mater. Sci. 29 (2) (1994) 294-323.

[3] Z. Bartczak, A. Galeski, Plasticity of semicrystalline polymers, Macromol. Symp. 294 (1) (2010) 67-90.

[4] J. Petermann, H. Gleiter, Direct observation of dislocations in polyethylene crystals, Philos. Mag. 25 (4) (1972) 813-816.

[5] H. Gleiter, A. S. Argon, Plastic deformation of polyethylene crystals, Philos. Mag. 24 (187) (1971) 71-80.

[6] A. Cowking, J. G. Rider, On molecular and textural reorientations in polyethylene caused by applied stress, J. Mater. Sci. 4 (12) (1969) 10511058 .

[7] F. C. Frank, A. Keller, A. O'Connor, H. H. Wills, Deformation processes in polyethylene interpreted in terms of crystal plasticity, Philos. Mag. 3 (25) (1958) 64-74.

[8] V. F. Holland, Dislocations in polyethylene single crystals, J. Appl. Phys. 35 (11) (1964) 3235-3241.

[9] R. J. Young, P. B. Bowden, J. M. Ritchie, J. G. Rider, Deformation mechanisms in oriented high-density polyethylene, J. Mater. Sci. 8 (1) (1973) 23-36.

[10] R. J. Young, P. B. Bowden, Twinning and martensitic transformations in oriented high-density polyethylene, Philos. Mag. 29 (5) (1974) 10611073.

[11] A. Galeski, Strength and toughness of crystalline polymer systems, Prog. Polym. Sci. 28 (12) (2003) 1643 - 1699.

[12] A. Galeski, Z. Bartczak, A. S. Argon, R. E. Cohen, Morphological alterations during texture-producing plastic plane strain compression of highdensity polyethylene, Macromol. 25 (21) (1992) 5705-5718.

[13] P. Allan, M. Bevis, Deformation processes in thin melt-cast films of highdensity polyethylene: II. Deformation processes in the non-equatorial regions of spherulites, Philos. Mag. A 41 (4) (1980) 555-572.

[14] M. Bevis, E. Crellin, The geometry of twinning and phase transformations in crystalline polyethylene, Polymer 12 (11) (1971) 666 - 684.

[15] P. Allan, E. B. Crellin, M. Bevis, Stress-induced twinning and phase transformations in polyethylene single crystals, Philos. Mag. 27 (1) (1973) $127-145$.

[16] P. Allan, M. Bevis, Deformation processes in thin melt-cast films of highdensity polyethylene: I. Experimental methods and deformation processes in the equatorial regions of spherulites, Philos. Mag 35 (2) (1977) 405-430.

[17] P. Allan, M. Bevis, Multiple deformation processes in polyethylene single crystals, Philos. Mag. 31 (5) (1975) 1001-1009.

[18] D. J. Bacon, N. A. Geary, Computer simulation of polyethylene crystals, part 1: Method, structure and elastic constants for perfect crystals, J. Mater. Sci. 18 (3) (1983) 853-863.

[19] N. A. Geary, D. J. Bacon, Computer simulation of polyethylene crystals, part 2: The boundary properties of twins and stacking faults, J. Mater. Sci. 18 (3) (1983) 864-883.

[20] D. J. Bacon, K. Tharmalingam, Computer simulation of polyethylene crystals, part 3: The core structure of dislocations, J. Mater. Sci. 18 (3) (1983) 884-893.

[21] D. C. Langreth, B. I. Lundqvist, S. D. Chakarova-Käck, V. R. Cooper, M. Dion, P. Hyldgaard, A. Kelkkanen, J. Kleis, L. Kong, S. Li, P. G. Moses, E. Murray, A. Puzder, H. Rydberg, E. Schröder, T. Thonhauser, A density functional for sparse matter, J. Phys.: Condens. Matter 21 (8) (2009) 084203.

[22] J. Kleis, B. I. Lundqvist, D. C. Langreth, E. Schröder, Towards a working density-functional theory for polymers: First-principles determination of the polyethylene crystal structure, Phys. Rev. B 76 (2007) 100201.

[23] H. Rydberg, M. Dion, N. Jacobson, E. Schröder, P. Hyldgaard, S. I. Simak, D. C. Langreth, B. I. Lundqvist, Van der Waals density functional for layered structures, Phys. Rev. Lett. 91 (2003) 126402.

[24] M. Dion, H. Rydberg, E. Schröder, D. C. Langreth, B. I. Lundqvist, Van der Waals density functional for general geometries, Phys. Rev. Lett. 92 (2004) 246401.

[25] M. Dion, H. Rydberg, E. Schröder, D. C. Langreth, B. I. Lundqvist, Erratum: Van der Waals density functional for general geometries [Phys. Rev. Lett. 92, 246401 (2004)], Phys. Rev. Lett. 95 (2005) 109902.

[26] T. Thonhauser, V. R. Cooper, S. Li, A. Puzder, P. Hyldgaard, D. C. Langreth, Van der Waals density functional: Self-consistent potential and the nature of the van der Waals bond, Phys. Rev. B 76 (2007) 125112.

[27] K. Berland, C. A. Arter, V. R. Cooper, K. Lee, B. I. Lundqvist, 
E. Schröder, T. Thonhauser, P. Hyldgaard, van der Waals density functionals built upon the electron-gas tradition: Facing the challenge of competing interactions, J. Chem. Phys. 140 (18) (2014) 18A539.

[28] P. Hyldgaard, K. Berland, E. Schröder, Interpretation of van der Waals density functionals, Phys. Rev B 90 (2014) 075148.

[29] T. Thonhauser, S. Zuluaga, C. A. Arter, K. Berland, E. Schröder, P. Hyldgaard, Spin signature of nonlocal correlation binding in metalorganic frameworks, Phys. Rev. Lett. 115 (2015) 136402.

[30] K. Berland, V. R. Cooper, K. Lee, E. Schröder, T. Thonhauser, P. Hyldgaard, B. I. Lundqvist, van der Waals forces in density functional theory: a review of the vdW-DF method, Rep. Prog. Phys. 78 (6) (2015) 066501.

[31] K. Berland, P. Hyldgaard, Exchange functional that tests the robustness of the plasmon description of the van der Waals density functional, Phys. Rev. B 89 (2014) 035412.

[32] I.-C. Yeh, J. L. Lenhart, G. C. Rutledge, J. W. Andzelm, Molecular dynamics simulation of the effects of layer thickness and chain tilt on tensile deformation mechanisms of semicrystalline polyethylene, Macromol. 50 (4) (2017) 1700-1712.

[33] V. Kumar, C. R. Locker, P. J. in't Veld, G. C. Rutledge, Effect of short chain branching on the interlamellar structure of semicrystalline polyethylene, Macromol. 50 (3) (2017) 1206-1214.

[34] X. Dong, D. L. McDowell, S. R. Kalidindi, K. I. Jacob, Dependence of mechanical properties on crystal orientation of semi-crystalline polyethylene structures, Polymer 55 (16) (2014) 4248 - 4257.

[35] N. Lempesis, P. J. in't Veld, G. C. Rutledge, Atomistic simulation of the structure and mechanics of a semicrystalline polyether, Macromol. 49 (15) (2016) 5714-5726.

[36] D. Hossain, M. Tschopp, D. Ward, J. Bouvard, P. Wang, M. Horstemeyer, Molecular dynamics simulations of deformation mechanisms of amorphous polyethylene, Polymer 51 (25) (2010) 6071 - 6083.

[37] I.-C. Yeh, J. W. Andzelm, G. C. Rutledge, Mechanical and structural characterization of semicrystalline polyethylene under tensile deformation by molecular dynamics simulations, Macromol. 48 (12) (2015) 4228-4239.

[38] J. M. Kim, R. Locker, G. C. Rutledge, Plastic deformation of semicrystalline polyethylene under extension, compression, and shear using molecular dynamics simulation, Macromol. 47 (7) (2014) 2515-2528.

[39] A. Ghazavizadeh, G. C. Rutledge, A. A. Atai, S. Ahzi, Y. Rmond, N. Soltani, Micromechanical characterization of the interphase layer in semi-crystalline polyethylene, J. Polymer Sci. B: Polymer Phys. 51 (16).

[40] S. Lee, G. C. Rutledge, Plastic deformation of semicrystalline polyethylene by molecular simulation, Macromol. 44 (8) (2011) 3096-3108.

[41] P. J. in't Veld, M. Htter, G. C. Rutledge, Temperature-dependent thermal and elastic properties of the interlamellar phase of semicrystalline polyethylene by molecular simulation, Macromol. 39 (1) (2006) 439-447.

[42] L. Ding, R. L. Davidchack, J. Pan, A molecular dynamics study of Young's modulus change of semi-crystalline polymers during degradation by chain scissions, J. Mech. Behav. Biomed. 5 (1) (2012) $224-230$.

[43] J. Kleis, E. Schröder, P. Hyldgaard, Nature and strength of bonding in a crystal of semiconducting nanotubes: van der Waals density functional calculations and analytical results, Phys. Rev. B. 77 (20) (2008) 205422.

[44] K. Lee, A. K. Kelkkanen, K. Berland, S. Andersson, D. C. Langreth, E. Schröder, B. I. Lundqvist, P. P. Hyldgaard, Evaluation of density functional with account of van der Waals forces by use of experimental $\mathrm{H} 2$ physisorption data on Cu(111), Phys. Rev. B 84 (2011) 193408.

[45] K. Lee, K. Berland, M. Yoon, S. Andersson, E. Schröder, P. Hyldgaard, B. I. Lundqvist, Benchmarking van der Waals density functionals with experimental data: potential-energy curves for $\mathrm{H} 2$ molecules on $\mathrm{Cu}(111)$ (100), and (110) surfaces, J. Phys.:Condens. Matter 24 (2012) 424213.

[46] K. Berland, P. Hyldgaard, Structure and binding in crystals of cagelike molecules: Hexamine and platonic hydrocarbons, J. Chem. Phys. 132 (13) (2010) 134705

[47] K. Berland, Ø. Borck, P. Hyldgaard, Van der Waals density functional calculations of binding in molecular crystals, Comp. Phys. Comm. 182 (9) (2011) 1800 - 1804

[48] K. Burke, Perspective on density functional theory, J. of Chem. Phys. 136 (2012) 150901

[49] A. D. Becke, Perspective: Fifty years of density-functional theory in chemical physics, J. Chem. Phys. 140 (2014) 18A301.

[50] G. Roman-Peres, J. M. Soler, Efficient implementation of a van der Waals density functional: Application to double-wall carbon nantubes, Phys.
Rev. Lett. 103 (2009) 096102.

[51] D. Le, A. Kara, P. Hyldgaard, E. Schröder, T. S. Rahman, Physisorption of nucleobases on graphene: a comparative van der Waals study, J. Phys.:Condens. Matter 24 (2012) 424210.

[52] A. H. Larsen, M. Kuisma, Y. Pouillon, J. Löfgren, P. Erhart, P. Hyldgaard, libvdwxc: a library for exchange-correlation functionals in the vdW-DF family, Model. Simul. Mater. Sci. Eng. (submitted).

[53] K. Lee, È. D. Murray, L. Kong, B. I. Lundqvist, D. C. Langreth, Higheraccuracy van der Waals density functional, Phys. Rev. B 82 (8) (2010) 081101.

[54] D. C. Langreth, J. P. Perdew, The exchange-correlation energy of a metallic surface, Sol. State Commun. 17 (1975) 1425.

[55] O. Gunnarsson, B. I. Lundqvist, Exchange and correlation in atoms, molecules, and solids by the spin-density-functional formalism, Phys. Rev. B 13 (1976) 4274.

[56] D. C. Langreth, J. P. Perdew, Exchange-correlation energy of a metallic surface: Wave-vector analysis, Phys. Rev. B 15 (1977) 2884.

[57] J. P. Perdew, K. Burke, M. Ernzerhof, Generalized gradient approximation made simple, Phys. Rev. Lett. 77 (1996) 3865-3868.

[58] L. Gharaee, P. Erhart, P. Hyldgaard, Finite-temperature properties of nonmagnetic transition metals: Comparison of the performance of constraintbased semilocal and nonlocal functionals, Phys. Rev. B 95 (2017) 087147.

[59] K. Rapcewizc, N. W. Ashcroft, Fluctuation attraction in condensed matter: A nonlocal functional approach, Phys. Rev B 44 (1991) 4032(R).

[60] T. Rangel, K. Berland, S. Sharifzadeh, F. Brown-Altvater, K. Lee, P. Hyldgaard, L. Kronik, J. B. Neaton, Structural and excited-state properties of oligoacene crystals from first principles, Phys. Rev. B 93 (2016) 115206.

[61] F. Brown-Altvater, T. Rangel, J. B. Neaton, Ab initio phonon dispersion in crystalline naphthalene using van der Waals density functionals, Phys. Rev. B 93 (2016) 195206.

[62] T. Björkman, Testing several recent van der Waals density functionals for layered structures, Journal of Chemical Physics 141 (7) (2014) 074708.

[63] P. Erhart, P. Hyldgaard, D. Lindroth, Microscopic origin of thermal conductivity reduction in disordered van der Waals solids, Chem. Mater. 27 (2015) 5511.

[64] D. O. Lindroth, P. Erhart, Thermal transport in van der Waals solids from first-principles calculations, Phys. Rev. B 94 (2016) 115205.

[65] J. Löfgren, H. Grönbeck, K. Moth-Poulsen, P. Erhart, Understanding the phase diagram of self-assembled monolayers of alkanethiolates on gold, J. Phys. Chem. C 120 (22) (2016) 12059.

[66] B. Borca, V. Schendel, R. Petuya, I. Pentegov, T. Michnowicz, , U. Kraft, H. Klauk, A. Arnau, P. Wahl, U. Schlickum, K. Kern, Bipolar conductance switching of single anthradithiophene molecules, ACS Nano 9 (2015) 12506

[67] K. Momma, F. Izumi, VESTA3 for three-dimensional visualization of crystal, volumetric and morphology data, J. Appl. Crystall. 44 (6) (2011) 1272-1276.

[68] Y. Kikuchi, S. Krimm, Infrared studies of the role of monoclinic structure in the deformation of polyethylene, J. Macromol. Sci.-Phys. B 4 (3) (1970) 461-472.

[69] V. Vitek, Intrinsic stacking faults in body-centred cubic crystals, Philos. Mag. 18 (154) (1968) 773-786.

[70] P. Giannozzi, S. Baroni, N. Bonini, M. Calandra, R. Car, C. Cavazzoni, D. Ceresoli, G. L. Chiarotti, M. Cococcioni, I. Dabo, A. Dal Corso, S. de Gironcoli, S. Fabris, G. Fratesi, R. Gebauer, U. Gerstmann, C. Gougoussis, A. Kokalj, M. Lazzeri, L. Martin-Samos, N. Marzari, F. Mauri, R. Mazzarello, S. Paolini, A. Pasquarello, L. Paulatto, C. Sbraccia, S. Scandolo, G. Sclauzero, A. P. Seitsonen, A. Smogunov, P. Umari, R. M. Wentzcovitch, Quantum espresso: a modular and open-source software project for quantum simulations of materials, J. Phys.: Condens. Matter 21 (39) (2009) 395502 (19pp).

[71] D. Vanderbilt, Soft self-consistent pseudopotentials in a generalized eigenvalue formalism, Phys. Rev. B 41 (1990) 7892-7895.

[72] K. F. Garrity, J. W. Bennett, K. M. Rabe, D. Vanderbilt, Pseudopotentials for high-throughput DFT calculations, Comput. Mater. Sci. 81 (2014) 446 -452 .

[73] H. J. Monkhorst, J. D. Pack, Special points for brillouin-zone integrations, Phys. Rev. B 13 (1976) 5188-5192.

[74] S. Plimpton, Fast parallel algorithms for short-range molecular dynamics, J. Comput. Phys. 117 (1) (1995) 1 - 19. 
URL http://lammps.sandia.gov

[75] G. Shrivastav, M. Agarwal, Stress-strain relationships in hydroxyl substituted polyethylene, J. Phys. Chem. B 120 (30) (2016) 7598-7605.

[76] W. L. Jorgensen, D. S. Maxwell, J. Tirado-Rives, Development and testing of the OPLS all-atom force field on conformational energetics and properties of organic liquids, J. American Chem. Soc. 118 (45) (1996) $11225-11236$.

[77] W. L. Jorgensen, J. Tirado-Rives, The OPLS [optimized potentials for liquid simulations] potential functions for proteins, energy minimizations for crystals of cyclic peptides and crambin, J. American Chem. Soc. 110 (6) (1988) 1657-1666.

[78] W. Paul, D. Y. Yoon, G. D. Smith, An optimized united atom model for simulations of polymethylene melts, J. Chem. Phys. 103 (4) (1995) 17021709.

[79] K. Bolton, S. B. M. Bosio, W. L. Hase, W. F. Schneider, K. C. Hass, Comparison of explicit and united atom models for alkane chains physisorbed on $\alpha-\mathrm{Al}_{2} \mathrm{O}_{3}$ (0001), J. Phys. Chem. B 103 (19) (1999) 3885-3895.

[80] P. J. in't Veld, G. C. Rutledge, Temperature-dependent elasticity of a semicrystalline interphase composed of freely rotating chains, Macromol. 36 (19) (2003) 7358-7365.

[81] S. Kavesh, J. M. Schultz, Lamellar and interlamellar structure in meltcrystallized polyethylene. I. Degree of crystallinity, atomic positions, particle size, and lattice disorder of the first and second kinds, J. Polym. Sci. A-2: Polym. Phys. 8 (2) (1970) 243-276.

[82] G. Avitabile, R. Napolitano, B. Pirozzi, K. D. Rouse, M. W. Thomas, B. T. M. Willis, Low temperature crystal structure of polyethylene: Results from a neutron diffraction study and from potential energy calculations, J Polym. Sci.: Polym. Lett. Ed. 13 (6) (1975) 351-355.

[83] M. S. Miao, M.-L. Zhang, V. E. V. Doren, C. V. Alsenoy, J. L. Martins, Density functional calculations on the structure of crystalline polyethylene under high pressures, J. Chem. Phys. 115 (24) (2001) 11317-11324.

[84] B. Montanari, P. Ballone, R. O. Jones, Density functional study of molecular crystals: Polyethylene and a crystalline analog of bisphenol-a polycarbonate, J. Chem. Phys. 108 (16) (1998) 6947-6951.

[85] P. W. Teare, The crystal structure of orthorhombic hexatriacontane, $\mathrm{C}_{36} \mathrm{H}_{74}$, Acta Crystall. 12 (4) (1959) 294-300.

[86] A. van de Walle, G. Ceder, Correcting overbinding in local-densityapproximation calculations, Phys. Rev. B 59 (1999) 14992-15001.

[87] K. Berland, P. Hyldgaard, Analysis of van der Waals density functional components: Binding and corrugation of benzene and C60 on boron nitride and graphene, Phys. Rev. B 87 (2013) 205421.

[88] J. F. Twisleton, J. W. White, P. A. Reynolds, Dynamical studies of fully oriented deuteropolyethylene by inelastic neturon scattering, Polymer 23 (4) (1982) 578 - 588.

[89] C. L. Choy, W. P. Leung, Elastic moduli of ultradrawn polyethylene, J. Polym. Sci.: Polym. Phys. Ed. 23 (9) (1985) 1759-1780.

[90] A. Kokalj, Computer graphics and graphical user interfaces as tools in simulations of matter at the atomic scale, Comput. Mater. Sci. 28 (2) (2003) $155-168$.

[91] B. I. Lundqvist, A. Bogicevic, K. Carling, S. V. Dudiy, S. Gao, J. Hardford, P. Hyldgaard, N. Jacobson, D. C. Langreth, N. Lorente, S. Ovesson, B. Razaznejad, C. Ruberto, H. Rydberg, E. Schröder, S. I. Simak, G. Wahnström, Y. Yourdshahyan, Density-functional bridge between surfaces and interfaces, Surf. Sci. 493 (2001) 253. 